\title{
DAS SEELSORGEKONZEPT DES IGNAZ HEINRICH VON WESSENBERG IN GRUNDZÜGEN
}

\section{Die Gestaltungsfaktoren der Persönlichkeit und die Ansichten über die Ekkle- siologie und die Seelsorge von Ignaz Heinrich von Wessenberg.}

Ignaz Heinrich von Wessenberg ${ }^{1}$ war einer der berühmtesten Reformatoren des kirchlichen Lebens in Baden-Württemberg im 19. Jahrhundert, der die Aufklärungsideale Josephs II auf den deutschen Boden übertragen wollte. Schon seit seiner Jugend war er wissbegierig und sehr stark von den josephinistischen Ideen geprägt. $\mathrm{Zu}$ Hause wurde er von seinem Vater - Phillip - in der Mentalität der österreichischen

1 Ignaz Heinrich Karl, Freiherr von Wessenberg (geb. am 4. November 1774 in Dresden; starb am 9. August 1860 in Konstanz) war ein aufgeklärter römisch-katholischer Theologe aus dem Adel. Sein Bruder war der österreichische Minister Johann von Wessenberg. Ignaz Heinrich Karl studierte Theologie in Augsburg, Dillingen, Würzburg und Wien. 1801 wurde er von Fürstbischof Karl Theodor von Dalberg zum Generalvikar des Bistums Konstanz ernannt, doch erst 1812 empfing er die Priesterweihe. Auf dem Wiener Kongress bemühte er sich in Dalbergs Auftrag um die Herstellung einer deutsch-katholischen Nationalkirche unter deutschem Primas. Infolgedessen versagte der Papst sowohl seiner Wahl zum Koadjutor 1814 wie zum Bistumsverweser 1817 die Bestätigung. Bis zur Auflösung des Bistums Konstanz 1821 schützte ihn die badische Regierung in der Ausübung seines Amtes. In der Folge gelang es nicht, ihn zum Erzbischof von Freiburg zu machen, da er vom Papst abgelehnt wurde. Seitdem lebte er in Konstanz als Privatmann und erhielt am 28. Juli 1832 die Ehrenbürgerrechte der Stadt Konstanz verliehen. Seine Gemäldesammlung stiftete er dem Großherzog Friedrich von Baden, der im Gegenzug 20.000 Gulden für wohltätige Zwecke gab. Der Großherzog bewilligte den Verbleib der Sammlung in Konstanz. Heute werden etwa 80 Gemälde zu dem der Zähringer Stiftung unterstehenden Bestand der Städtischen Wessenberg-Galerie gerechnet. Wessenberg verfasste insgesamt rund 470 Publikationen und war Mitarbeiter der Freymüthigen Blätter über Theologie und Kirchenthum. Seine umfangreiche Privatbibliothek von über 20.000 Bänden vererbte er der Stadt Konstanz, die sie pflegte und erweiterte. Zudem stiftete er die Wessenberganstalt. Seit dem Jahr 2000 ist die Wessenberg-Bibliothek als geschlossener Buchbestand in der Bibliothek der Universität Konstanz für Forschungszwecke zugänglich. In Erinnerung an die Person sind in Konstanz die Straße an dem ehemaligen Wohnhaus sowie ein Wirtschaftsgymnasium nach Wessenberg benannt. K.H. Braun, Wessenberg, Ignaz Heinrich von (1774-1860), in: Die Bischöfe der deutschsprachigen Länder, 1785/1803 bis 1945. Ein biographisches Lexikon, Hg. E. Gatz, Berlin 1893, S. 808-812. 
Aufklärung erzogen. Phillip Wessenberg war ein Konferenzminister ${ }^{2}$ und ein ehemaliger Prinzenerzieher am Katholischen Dresdener Hof und hat seinem Sohn Ignaz und seinen Geschwistern das gesellschaftlich moralische Miteinander und eine binnenkirchliche Pietät beigebracht ${ }^{3}$. Diese äußerst strenge intellektuelle Erziehung konnte man schon sehr früh im Leben des jungen Wessenberg beobachten. Sehr deutlich zeigen es die Erinnerungen aus seiner Jugendzeit, die er auf Papier hintergelassen hat. In seinem Tagebuch schrieb er unter anderem über sein Studium der Philosophie im ehemaligen Jesuitenkolleg St. Salvator in Augsburg, wo er ganz offen das Schulwesen kritisiert hat. Dort bemängelte er zum Beispiel die Vernachlässigung der deutschen Sprache in der Literatur ${ }^{4}$. Er schreibt: „Der Unterricht in der Geschichte beschränkte sich auf mechanisches Einlernen der Nahmen und Jahreszahlen, Schlachten und Todfähllen"s. Die bereits zu Hause erfahrene Ausbildung erhält Ignaz Heinrich ebenfalls an den Universtäten in Dillingen, Würzburg und Wien, welche die Aufklärungsideale Josephs II vertreten haben ${ }^{6}$. Vor allem in Dillingen hat er am praktischen Studium der Hl. Schrift, der Patrologie, der Konzilien, der Liturgie, des Pastorals und der Moral teilgenommen. Dort hat Wessenberg auch die enge Verknüpfung von Bibeltheologie und Glauben von dem damals in Dillingen tätigen Johann Michael Seiler übernommen ${ }^{7}$. Diese aufgeklärten Ideale haben sich im ganzen Leben des Wessenberg abgebildet. Sie haben ihn geprägt.

2 K.H. Braun, Ganz diesem Beruf zu leben... mein volles Kraftmaß zu widmen..., in: Glauben gestalten, Glaubensgestalten: mit Robert Zollitsch auf dem Weg; zum 70. Geburtstag des Erzbischofs von Freiburg und Vorsitzenden der Deutschen Bischofskonferenz, Hg. F. Deck, Freiburg 2008, S. 226.

3 K.H. Braun, Die Lebensgeister der Kirche, Glaube und Liebe, bedürfen, um stets ungeschwächt und ungestört zu wirken, der beständigen Erneuerung. Zum Kirchenbild Ignaz Heinrich von Wessenbergs, in: Kontinuität und Innovation um 1803. Säkularisation als Transformationsprozeß. Kirche, Theologie, Kultur, Staat, Hg. R. Decot, Mainz 2005, S. 24.

4 I.H. Wessenberg, Unveräffentlichte Manuskripte und Brieffe I/1, Hg. K. Aland, V. Müller, Freiburg-Basel-Wien 1968, S. 20.

5 I.H. Wessenberg, Unveräffentlichte Manuskripte..., op. cit., S. 178.

6 K.H. Braun, Den Glauben bewusster leben: vor 150 starb der Aufklärer Ignaz Heinrich von Wessenberg, „Konradsblatt” 2010, Nr. 32, S. 21.

7 K.H. Braun, Wessenberg, Ignaz Heinrich..., op. cit., S. 808. Johann Michael Sailer, ab 1826 von Sailer- geb. am 17. November 1751 in Aresing, Oberbayern, starb am 20. Mai1832 in Regensburg. Ein katholischer Theologe (insbesondere Pastoraltheologe) und Bischof von Regensburg. Er machte 1770 seinen Gymnasialabschluss am Jesuitengymnasium München (heute Wilhelmsgymnasium München) und begann dann als Novize bei den Jesuiten (bis zur Auflösung des Ordens 1773) ein Theologiestudium an der Universität Ingolstadt. Am 23. September 1775 empfing er in Augsburg die Priesterweihe. Als Repetitor (ab 1777) und Professor der Dogmatik (ab 1780) lehrte er in Ingolstadt, bis er 1781 unter dem Vorwurf des „Obskurantismus“ entlassen wurde.1784 erhielt er eine Professur für Theologie an der Universität Dillingen. Sailer wirkte als Buchautor und als Lehrer. Er 
Wenn man Wessenbergs Seelsorgekonzept, das sehr stark an die josephinische Aufklärung anknüpfte, beleuchten möchte, sollte man seine Lebenswirklichkeit in einer kulturgeschichtlichen Fragestellung betrachten. Hier muss man nach der Kraft der josephinischen Reformen, die auch die Feier der Gottesdienste und die emotionale Seite der Gläubigen beeinflusst haben, fragen ${ }^{8}$. In der Barockzeit wollte man die Emotionalität und Affekte in Richtung Religiosität anregen. „Es ging um notwendige Erfahrungshorizonte christlicher Askese, eines geistlichen Kampfes, der auf die konkrete Beherrschung von Körper und Geist, von Welt schlechthin abzielt"'. Den Gläubigen war es damals wichtig, Selbstkontrolle, Selbstregulierung oder Selbstmodellierung zu leben ${ }^{10}$. Die Seelsorger, die im Geist der josephinischen Aufklärung gearbeitet haben, bemerkten, dass sich die Bedürfnisse der Menschen im Laufe der Zeit gründlich verändert haben.

An dieser Stelle ist es sinnvoll, Beispiele einiger josephinischen Bischöfe bezüglich ihrer Ansichten und Seelsorge kurz darzustellen, um Wessenbergs Seelsorgebild und seine Beziehung zur österreichischen Aufklärung besser zu verstehen.

Als erstes Beispiel dient Leopold Ernst Graf von Firmian aus Passau ${ }^{11}$. Er war ein Berater der Kaiserin Maria Theresia, welcher er Anstoß zu der Schulreform in den Jahren 1769-1774 gegeben hat. Es ging um die Einführung der allgemeinen Schulpflicht. Zugleich richtete er in Passau ein Seminar zur Ausbildung der Welt-

war der Begründer und Hauptvertreter einer innerlichen und dabei duldsamen Richtung innerhalb der katholischen Kirche und zählt zu den Pionieren der Pastoraltheologie. Er wurde 1821 in Regensburg erster Domkapitular,1822 Generalvikar, Koadjutor und Titularbischof von Germanicopolis. Die Bischofsweihe empfing er am 28. Oktober 1822 vom Münchener Erzbischof Lothar Anselm von Gebsattel. 1825 wurde Sailer Dompropst am Regensburger Dom und endlich am 28. Oktober 1829 Bischof von Regensburg. Einer seiner engsten Mitarbeiter in Regensburg war Melchior von Diepenbrock. Nach mehreren Schlaganfällen starb Sailer 1832 in Regensburg. F.H. Reusch, Sailer Johann Michael von, in: Allgemeine Deutsche Biographie, Bd. 30, Leipzig 1890, S. 178-192.

8 K. H. Braun, Wieder die Säkularisation. Obrigkeitliche Steuerungen von Religiosität innerhalb »josephinischer« Kirchenordnungen, [In:] Aufmerksame Solidartät: Festschrift für Bischof Maximilian Aichern zum 70. Geburtstag, Hg. P. Hofer, Regensburg 2002, S. 13-14.

9 K.H. Braun, (Liberale) Kritik am Mönchtum, in: Mittelalterliches Mönchtum in der Moderne? Die Neugründigung der Benediktionerabtei Beuron 1863 und deren kulturelle Ausstrahlung im 19. und 20. Jahreshundert, Hg. W. Schöntag, Stuttgart 2015, S. 87.

10 K.H. Braun, Wieder die Säkularisation..., op. cit., S. 14.

11 Leopold Ernst Graf von Firmian (22.09.1708 - 13.03.1783) geboren in Trient, war Fürstbischof von Passau und Kardinal. Früher schon mit 31 Jahren Bischof von Seckau und war darüber hinaus zehn Jahre Administrator des Suffraganbistums Trient Er gilt einerseits als der letzte „Barockfürst” auf dem Passauer Bischofsthron, andererseits sind ihm auch richtungsweisende Entscheidungen als geistlicher wie weltlicher Herrscher zu verdanken.

A. Leidl, Leopold Ernst von Firmian, in: Neue Deutsche Biographie, Bd. 14, Berlin 1985, S. 293. 
priester ein. Dort war er Autor der Statuten für die Priesterbildung, die nicht nur theologisch orientierte Quellen enthielten, sondern auch aszetische Übungen und Haltungen der zukünftigen Priester prägten ${ }^{12}$. Firmian zeichnete sein Priesterbild nach „Pastor Bonus” von Jan von Opstraets. Gott war für ihn der gütige himmlische Vater, der uns erschaffen und als seine Kinder angenommen hat. Laut Firmian hat Gott eine besondere Sorgfalt und väterliche Liebe für uns ${ }^{13}$.

Hieronymus Joseph Franz de Paula Graf von Colloredoaus Salzburg ${ }^{14}$ - ein zweites Beispiel - hat ebenfalls Wessenberg einen Hinweis auf die Priesterausbildung gegeben. Nach der Meinung Colloredos sollte den Platz kasuistischer Spekulationen eine biblische und historische Theologie einnehmen und die ganze Priestererziehung stringent auf die Seelsorgepraxis ausgerichtet werden. Von seinen Priestern forderte er beispielsweise: mehr Liebe, Sanftmut, Freundlichkeit und Leutseligkeit. Sie sollten Gott so liebenswürdig darstellen, dass die Menschen Diesem vollkommen vertrauen könnten. Colloredo bezeichnet Gott u.a. auch als den weisesten und gütigsten Herrscher der Welt, der unendlich reich, glücklich und bedürfnislos ist und mit seiner Liebe und Fürsorge die ganze Welt umfasst. Das Ziel solch eines Gottesbildes war es, die Gläubigen vom Abfahren auf die Heilige Maria und andere gewisse Heilige »als leicht bestechenbare und feile Advokaten« abzuhalten. Für Colloredo war es wichtig, auf dem Gebiet der Liturgie eine »nur mechanisch handwerksmäßige« Oberflächlichkeit zu vermeiden. Er hat „Herzensreligion” dem „gedankenlosen Mitmachen leerer Zeremonien" entgegengestellt ${ }^{15}$. Auch er war also ein Verfechter von Reformen im Sinne der Aufklärung. Colloredo verbot unter anderem die Wassertaufe der Metzgergesellen (Metzgerspringen), das Wetterläuten, das Mitführen von lebenden Bildern, das Abschießen von Böllern bei Prozessionen und die Eselsritte am Palmsonntag. 1779 kritisierte er auch die Passionsspiele ${ }^{16}$.

12 A. Leidl, Leopold Ernst Kardinal von Firmian, „Ostbairische Grenzmarken. Passauer Jahrbuch für Geschichte, Kunst und Volkskunde" 1971, Bd. 13, S. 12-13.

13 K.H. Braun, Wieder die Säkularisation ..., op. cit., S. 18-21.

14 Hieronymus Franz de Paula Josef Graf Colloredo von Waldsee und Mels, auch Wallsee, (31.05.1732 - 20.05.1812) geboren in Wien, war 1761-1772 Bischof von Gurkund 17721803 der letzte regierende Fürsterzbischof des Fürsterzbistums Salzburg, danach noch von Wien aus bis zu seinem Ableben geistliches Oberhaupt der Erzdiözese Salzburg. Colloredo wirkte und handelte als ein ausgeprägter Vertreter der Katholischen Aufklärung. Er war einer der rund 1500 ermittelten Mitglieder des Illuminatenordens. J.Ch. Allmayer-Beck, Colloredo-Waldsee, Hieronymus Graf von, in: Neue Deutsche Biographie, Bd. 3, Berlin 1957, s. 327; Vgl: F.W. Bautz, Colloredo Hieronymus Graf von, in: Biographisch-Bibliographisches Kirchenlexikon, Bd. 1, Hamm 1990, Sp. 1098.

15 K.H. Braun, Wieder die Säkularisation ..., op. cit., S. 18-23.

16 J.Ch. Allmayer-Beck, Colloredo-Waldsee..., op. cit., S. 327; vgl: F.W. Bautz, Colloredo Hieronymus..., op. cit., Sp. 1098. 
Das dritte Beispiel widmet sich Joseph Anthon Gall von Linz ${ }^{17}$. Er war seit 1780 Oberaufseher über alle deutschen Schulen in Niederösterreich. Für sein Handeln wurde er schließlich zum Bischof ernannt ${ }^{18}$. Joseph II unterstützte die Diözese Linz, deren Staats- und Religionspolitik für Josephinismus bekannt wurde. Als behutsamer Aufklärer wandte er sich gegen abergläubische Praktiken und gründete 1802 die Theologisch-praktische Monatsschrift, in der er auch selbst publizierte. Des Weiteren gründete Gall 1806 das Linzer Priesterseminar seiner Diözese ${ }^{19}$. Seine bischöfliche Aufgabe sah er darin, sowohl die Motivation der Geistlichen zu stärken, als auch den Büchererwerb und die Weiterbildung durch finanzielle Besserstellung zu ermöglichen, sodass unabhängige Seelsorge ausgeübt werden konnte. So ausgebildete Geistliche, die ihren Dienst nicht nur nach Vorschrift oder bloßer Einhaltung von Riten und Gebräuchen ausübten, sollten ein Vorbild für alle Gläubigen werden. Nach Gall sollten die Priester Gott als liebevoll sorgenden Vater verstehen, der sich uns, Menschen, in der Person des Jesus Christus zugewandt hat. Die Menschen sollen alles tun, um Gott zu gefallen, denn Gottes Wirken kann nur dort geschehen, wo der Mensch seinen Beitrag leistet. Andererseits ist es ein „faules Gebet, welsches Gott nicht erhöret"20.

Als Zentrum der südwestdeutschen katholischen Aufklärung zwischen Josephinismus und Frühliberalismus galt u.a. die Stadt Freiburg im Breisgau, wo die praktischen Reformen der Seelsorge und der Liturgie durchgeführt wurden. Zuerst versuchte man die Muttersprache in der Katechetik durchzusetzen, um Theologie stärker an die modernen Wissenschaften anzupassen, Wallfahrten, Heiligenund Reliquienverehrung, sowie Ablasswesen dagegen einzuschränken ${ }^{21}$. All diese oben genannten Beispiele der aufgeklärten Ideen sind im ganzen priesterlichen Tun Wessenbergs sowie in seinen Ansichten und in seiner Lehre deutlich sichtbar und spürbar.

17 Joseph Anton Gall (27.03.1748 - 18.06.1807) geboren in Weil der Stadt, war katholischer Geistlicher und Pädagoge. 1773 bildete er sich bei dem Reformator des österreichischen Bildungswesens. Abt. Am 22. April 1787 wurde er als Domscholaster des Wiener Dom und Metropolitankapitels investiert. 1788 wurde er duch Kaiser Joseph II. als Nachfolger Ernest Johann Nepomuks von Herberstein zum zweiten Diözesanbischof der 1784 gegründeten Diözese Linzernant, was Papst Pius VI. am 15. Dezember 1788 bestätigte. H. Ferihumer, Gall Joseph Anton, in: Neue Deutsche Biographie, Bd. 6, Berlin 1964, S. 42.

18 K.H. Braun, Wieder die Säkularisation ..., op. cit., S. 17.

19 K. Werner, Gall Joseph Anton, in: Allgemeine Deutsche Biographie, Bd. 8, Leipzig 1878, S. 317; Vgl: H. Ferihumer, Gall, Joseph..., op. cit., S. 42.

${ }^{20}$ K.H. Braun, Wieder die Säkularisation ..., op. cit., S. 18-22.

${ }^{21}$ A. Aurnhammer, B. Besslich, Freiburg als Zentrum der südwestdeutschen katholischen Aufklärung zwischen Josephinismus und Frühliberalismus, in: Der Ort und das Ereignis: die Kulturzentren in der europäischen Geschichte, Hg. A. Venturelli, Freiburg 2002, S. $122-123$. 


\section{Die persönliche ekklesiologische und seelsorgliche Ansichten von Ignaz Hein- rich von Wessenberg.}

\section{1) Gottesbild}

Ignaz Heinrich von Wessenberg ging in seinem Verständnis der Religiosität von einem biblischen Gottesbild aus. Trinität und Inkarnation waren für ihn von groBer Bedeutung. Der laut ihm fürsorgende Gott ist der Welt zugewandt und ist den Menschen sehr nahe. Er ist kein deistischer Gott, wie es manche Aufklärer behaupteten, sondern ein theistischer, der seine »Vorsicht« und seine »Vorsehung« über den Menschen walten lässt. Nach der Meinung Wessenbergs kann man Gott dann begegnen, wenn man seinen eigenen Verstand der Gabe Gottes und der Offenbarung Jesu Christi gegenüber richtig einsetzt. Gott hat den Menschen nicht nur geschaffen, Er hält auch seine Existenz im Gang. Wessenbergs Gottesbild war dynamisch. Der handelnde Gott bewegt nämlich unser Denken und unsere Existenz. Nach der Logik des Konstanzer Generalvikars gehen »Leben und Bewegung « dem Denken und dem Sein voraus. Er hat auch darauf hingewiesen, dass in der Beziehung zwischen Gottes Zuwendung in Jesus Christus und der entsprechenden Einstellung der Menschen eine von Gott geschenkte Freiheit »das Böse zu beseitigen und das Gute zu vollbringen« kann. Was noch bei Wessenbergs Gottesbilds wichtig erscheint, ist die Tatsache, dass Christus seine Lehre nicht buchstäblich hinterlassen hat. Der Konstanzer Generalvikar wollte die kirchliche Botschaft im christlichen Geist der Liebe und des Dienstes vermitteln und bezieht sich deshalb auf die biblische Botschaft: „Der Buchstabe tödtet, nur der Geist belebt!"’22.

\section{2) Dogmatik}

Da von der kirchlichen Dogmatik die religiöse Vitalität abhängt, wurde diese von Ignaz Heinrich von Wessenberg selbstverständlich akzeptiert. Er interessierte sich am Meisten für die Übermittlung dieser und hier bewegt sich der Generalvikar innerhalb der pastoralen Tradition josephinischer Bischöfe, ähnlich wie die schon oben genannten Colloredo, Gall und Firmian. Die dogmatische Frage vermied Wessenberg jedoch im Bereich der Seelsorge ${ }^{23}$. Seine Theologie wurde im Rahmen katholischer Selbstverständlichkeiten erfasst. Christus war für ihn ein echter Gott und ein echter Mensch, für jene, die an Ihn glauben, soll Er der Mittelpunkt des Lebens sein ${ }^{24}$.

\section{3) Ekklesiologie}

Ignaz Heinrich von Wessenberg besaß keine eigene systematische Ekklesiologie, die man leicht beschreiben könnte. Sein Kirchenbild entwickelte sich parallel zu seiner steigenden Lebenserfahrung. Er wollte seine Theologie stets erweitern und

22 A. Aurnhammer, B. Besslich, Freiburg als Zentrum ..., op. cit., S. 25-33.

23 K.H. Braun, Den Glauben bewusster..., op. cit., S. 21.

${ }^{24}$ K.H. Braun, Der Gottsucher..., op. cit., S. 33. 
veredeln, damit sie humaner wird und somit das Gehirn und Gemüt nicht vertrocknen lässt. Der Konstanzer Generalvikar stellte fest, dass die Theologie nur durch die Dynamik der Praxis ihren Wert erhalten kann. Nach seiner Ekklesiologie waren das Gespür und die Fähigkeit, die jeweiligen Zeitbedürfnisse und ihre gesellschaftliche Komplexität in Betracht zu setzen, sehr wichtig. Der Konstanzer Generalvikar war stark davon überzeugt, dass nur die katholische Religion, die von Jesus Christus gestiftet worden ist, wahr sei, und dass die kirchlichen Sakramente in den Gläubigen zu „kräftigen, sichtbaren Zeichen der innerlich wirkenden göttlichen Gnade” würden ${ }^{25}$. Zugleich jedoch war für Wessenberg die katholische Kirche auch jene, auf die sich die protestantischen Konfessionen berufen konnten und deswegen sollten sich auch diese auf einer staatlichen Ebene gut verstehen. Der Konstanzer Generalvikar hat dennoch betont, dass der katholische Glaube an der Wahrheit orientiert ist ${ }^{26}$.

Bemerkenswert ist hier auch Wessenbergs Verhältnis zum Papsttum. Er wollte, dass dieses nicht auf eine gerichtliche Art und Weise verstanden wird, sondern viel mehr als eine geistliche Autorität ${ }^{27}$. Seiner Meinung nach müsste das Papsttum eine geistliche Qualität darstellen ${ }^{28}$. Jedoch wäre solch eine Qualität nicht als eine Frage der Unfehlbarkeit des Papstes zu verstehen. Wessenberg behauptete, dass von solcher Qualität in weltlichen und politischen Angelegenheiten gar keine Rede sein könnte $^{29}$. Viel wichtiger als das Papstamt waren für den Konstanzer Generalvikar die Konzilien und Synoden, wo - seiner Meinung nach - auch die Laien zu Wort kommen sollten ${ }^{30}$. Interessant ist sein Blickpunkt auf die Bischöfe und ihre Pflichten. Und zwar sollten diese nicht nur Vorbilder einer tugendhaften Lebensweise für andere Menschen sein, sondern auch auf eine aufgeklärte Weise das Gemeinwohl fördern. Ein wichtiges Merkmal aller Bischöfe war damals auch die Verwaltungsfähigkeit. Sie alle sollten ihren Grundeigentum gut verwalten ${ }^{31}$. Über die Priester wiederum hat der Konstanzer Generalvikar geschrieben, dass sie keine Herren des Glaubens sein sollten, denn dies wäre die Rolle ihrer aristokratischen Vermittler ${ }^{32}$. Des Weiteren war für Wessenberg das Heranbilden des Klerus eine Herzensangele-

25 K.H. Braun, Die Lebensgeister der Kirche..., op. cit., S. 24-25.

${ }^{26}$ K.H. Braun, Die Lebensgeister der Kirche..., op. cit., S. 35.

${ }^{27}$ I.H. Wssenberg, Die Eintracht zwischen Kirche und Staat, Hg. und Vorw. J. Beck, Aarau 1869, S. VIII.

28 K.H. Braun, Ganz diesem Beruf..., op. cit., S. 230-231.

29 I. H. Wssenberg, Die Eintracht zwischen..., op. cit., S. 202; Vgl: K.H. Braun, Kirche im liberalen Bürgerstaat Das Erzbistum von der Mitte des 19. Jahrhunderts bis zum Ende der Monarchie 1918, In: Geschichte der Erzdiözese Freiburg Freiburg, Bd. 1, Hg. H. Smolinsky, Freiburg im Breisgau 2008, S. 170.

30 B. Schmidt, Die Konzilien und der Papst. Von Pisa (1409) bis zum Zweiten Vatikanischen Konzil (1962-65), Freiburg im Breisgau 2013, S. 213.

31 K.H. Braun, Die Lebensgeister der Kirche..., op. cit., S. 26-27.

32 K.H. Braun, Den Glauben bewusster..., op. cit., S. 21. 
genheit $^{33}$. Eine Beschreibung des Großteils seines Seelsorgekonzepts wird noch in dieser Dissertation folgen.

In der Ekklesiologie Wessenbergs haben wir auch mit der Frage nach dem Stellenwert des (monastischen) Ordenslebens zu tun. Der Konstanzer Generalvikar war ein sehr praktisch denkender Mensch und wollte in der Tat nicht zu dem vorsäkularen kontemplativen Orden zurückkehren. Von Bedeutung waren für ihn nur jene Brüder und Schwestern, die die Kranken und Schwachen pflegten, und jene, die sich der Erziehung und dem Unterricht der Kinder widmeten. Die anderen Orden sollten den Schwerpunkt ihrer Entwicklung auf Verhandlungen mit den Bundesstaaten legen ${ }^{34}$.

Sehr interessant ist hier auch die Staatskirchenfrage. Wessenberg schätzte die Religiosität als ein gesellschaftliches Wohlergehen der selbstverständlichen Wertschätzung und Sicherung von kirchlicher und staatlicher Autorität ein ${ }^{35}$. Die für ihn offensichtliche Voraussetzung, dass die Religion die haltbarste Grundlage des Staates und seines Wohlstands sei, sowie der Anspruch auf die Beseitigung der Säkularisation mit all ihren Folgen oder die Forderung von Bundeskonkordat bedeuteten nicht, dass er eine politisch selbstständige Nationalkirche begründen wollte. Sein Ziel war es, alle deutschen Diözesen unter die Leitung eines Primas zu stellen ${ }^{36}$. Im Jahre 1848 hat er geschrieben, dass die echte Freiheit der Kirche „von ihr selbst, von ihrem Streben nach Heiligkeit auszugehen muss ${ }^{\prime 37}$. Wessenberg hat bemerkt, dass die Kirche, die von Staat und Gesellschaft distanziert wurde, in eine Einseitigkeit kommen könnte und sich dadurch das politische, das bürgerliche und das religiöse Leben voneinander ganz entfremden könnten. Er dachte sogar, dass die Kirche für den Staatsaufbau elementare Funktionen wahrnehmen könne, stattdessen bedürfe sie aber eines staatlichen Schutzes und einer gewissen Privilegierung ${ }^{38}$.

Das allgemeine Kirchenbild ist bei Wessenberg christozentrisch gedacht. Die Kirche sollte nicht institutionell definiert werden, sondern als jene, die in der gesellschaftlichen Kultur die bürgerliche Tugendhaftigkeit propagiert und sichert ${ }^{39}$. Die

33 S. Schmid, Der Priester:»In der Mitte zwischen Gott und den Menschen, wischen der Gemeinde und dem Altar«, in: Bildung bei Ignaz Heinrich von Wessenberg (1774-1860), Hg. K.H. Braun, Freiburg im Breisgau 2014, S. 39.

${ }^{34}$ K.H. Braun, Die Lebensgeister der Kirche..., op. cit., S. 28-29; Vgl: C. Nörber, Wessenberg Ignaz Heinrich von, http://kathenzyklo.bplaced.net/artikel.php?artikel=wessenberg [Zugang: 25 X 2019].

35 K.H. Braun, Kirche im liberalen ..., op. cit., S. 129.

36 F.X. Bischof, »Die Einheit der Nationalkirche schien mir zunächst das Wesentliche, wenn sich das religiös-kirchliche Leben Unseres Volkes haben und gedeihlich sich entwickeln soll«. Wessenberg auf dem Wiener Kongress, in: Der Wiener Kngress - eine kirchenpolitische Zänsur?, Hg. H. Duchhardt, J. Wischmeyer, Göttingen 2013, S. 105.

37 K.H. Braun, Die Lebensgeister der Kirche..., op. cit., S. 32.

38 M.Bangert, Bild und Glaube. Ästhetik und Siritualität bei Ignaz Heinrich von Wessenberg (1774-1860), Stuttgart 2009, S. 376.

39 K.H. Braun, Die Lebensgeister der Kirche..., op. cit., S. 36. 
Kirche sollte der ganzen Welt auf die ewige sittliche Weltordnung hinweisen, sodass der/die Einzelne sich stets »dem Gemeinwohl« unterordnet ${ }^{40}$.

\section{Die verwirklichte Ekklesiologie auf dem Gebiet der Seelsorge von Ignaz Heinrich von Wessenberg.}

Erziehung und Seelsorge waren für die Ekklesiologie Wessenbergs der „Sitz im Leben”41. Wessenberg war ein Aufklärer. „In der katholischen Aufklärung am Ende der Barockzeit hieß das konkret als die Reduktion von Äußerlichkeiten in den Bereichen einer Populärkultur und die Intensivierung jener Gottesdienste, die zugleich Lebensdienste sind"42. Für Wessenberg war dies jedoch zu wenig. Er wollte, dass die Gläubigen ihren Glauben bewusster erleben. Die Begriffe »Aufklärung« und »Bildung « waren für den Konstanzer Generalvikar nicht nur miteinander stark verbun$\mathrm{den}^{43}$. Man sagt, dass für Wessenberg die Lernfähigkeit ein Zeichen eines gläubigen Christen sei. Bei der Beziehung zwischen Gott und Mensch war nicht nur das Gefühl wichtig, sondern vor Allem das Nachdenken darüber, von Ihm erfüllt zu werden. Für Wessenberg war eine Wechselwirkung zwischen Fühlen und Denken bedeutsam, das Denken war jedoch im Bereich der Religion wichtiger ${ }^{44}$. Gott kann nur dann aufbauen, wenn Er Raum in unserem Denken finden kann. Dann kann Gott auch wirklich zu einem Gestaltungsfaktor in uns werden ${ }^{45}$.

\section{1) Priesterbildung}

Wessenberg sah in der Priesterbildung den Schlüssel für einen allmählichen Wandel der katholischen Kirche ${ }^{46}$. Aussicht auf Erfolg könnte nur dann bestehen, wenn der Klerus sich für diese öffne und sich dem Bistum zuverlässige Mitarbeiter zur Verfügung stellten ${ }^{47}$. Für ihn waren Priester nicht nur Liturgen oder Spender der Sakramente, sondern auch u.a. Lehrer ${ }^{48}$. Deswegen wusste er genau, dass nur gute Hirten

${ }^{40}$ K.H. Braun, Der Gottsucher: Wie stellt er die Gottesfrage?, in: Bildung bei Ignaz Heinrich von Wessenberg (1774-1860), Hg. K.H. Braun, Freiburg im Breisgau 2014, S. 25.

${ }^{41}$ K.H. Braun, Die Lebensgeister der Kirche..., op. cit., S. 24.

42 K.H. Braun, Den Glauben bewusster..., op. cit., S. 20.

43 D. Rimmale, Der Bildung des Voskes: Die Bildungspolitik Wessenbergs, in: Bildung bei Ignaz Heinrich von Wessenberg (1774-1860), Hg. K.H. Braun, Freiburg im Breisgau 2014, S. 43.

${ }^{44}$ K.H. Braun, Die Lebensgeister der Kirche..., op. cit., S. 29-30.

45 K.H. Braun, Der Gottsucher..., op. cit., S. 35.

46 S. Ruppert, Kirchenrecht und Kulturkampf. Historische Legitimation, politische Mitwirkung und wissenschaftliche Begleitung durch die Schule Emil Ludwig Richters, Tübingen 2002, S. 101.

47 F.X. Bischof, Die Bemühungen des Konstanzer Generalvikars Ignaz Heinrich von Wessenberg um die Priesterfortbildung, S. 103, https://mthz.ub.unimuenchen.de/MThZ/article/download/1995H1S99-117/3938/ [Zugang: 27 X 2018].

48 S. Schmid, Der Priester: »In der Mitte..., op. cit., S. 37. 
imstande sind, eine gute Herde zu erziehen. Für ihn waren die Priester Multiplikatoren, die das eigene Wissen an die Bevölkerung weitergeben sollten ${ }^{49}$.

1801 wurde der Konstanzer Generalvikar mit einer Seminarvisitation in Meersburg beauftragt. Dort sprach er zu den Priesterseminaristen: „Je besser der Klerus aus dem Seminar hervorgehe, desto bessere Priester werde er selbst in »seinem" Bistum haben". Wichtig für Wessenberg war ein geregeltes und geordnetes Seminarleben. Seiner Meinung nach sollten die Alumnen größtenteils an den Universitäten und Lyzeen studieren, um das Gedankengut der Aufklärung zu rezipieren. Im Jahre 1803 hat er den Seminarstatut geschaffen; dort hat Wessenberg beispielsweise etwas über ,eine stärkere praxisorientierte Ausbildung [geschrieben], darunter sei besonderes die Predigtübung hervorzuheben, aber auch die gewünschte Verwandlung der Muttersprache neben Latein innerhalb der Liturgie, z.B. ein regelmäßig gesungenes deutsches Amt im Priesterseminar, Hinführung zur Pfarrseelsorge, darunter das Verbot der Wallfahrt und der damit verbundenen Beichte, der regelmäßige Empfang der Sakramente und die Forderung nach sittlich korrektem Verhalten". Vor den Weihekandidaten hielt er auch sehr geistig-geistlich orientierte Sprachen, wo er sein Gottes- und Priesterbild vermittelt hat ${ }^{50}$.

Laut Wessenberg sollten sich die zukünftigen Priester intensiv mit der Bibel - vor allem mit dem Neuen Testament - und der Kirchengeschichte beschäftigen. Dazu sollte ein starker Akzent auf die Selbsterziehung und permanentes Lernen gelegt werden, damit die Priester mit eigenen Reflexionen und Erfahrungen vor den Gläubigen auftreten könnten. Darum müssen sie sich über ihr kirchliches Leben kümmern, indem sie es durch die Lektüre guter Bücher auf dessen Erneuerung bewirken. Wessenberg ist zu dem Entschluss gekommen, dass jeder Priester vorbildlich in seinem Benehmen sein sollte. Er dürfe nicht rauchen, keine Wirtshäuser oder Theater besuchen, nur einfache, dunkle und saubere Kleidung (keine Klerikerkleidung) tragen ${ }^{51}$. Man könnte sagen, dass Wessenberg keine ungehorsamen Priester haben wollte. In seinen Schriften findet man solche Worte: ,lieber gar keine Geistlichen als geistesträge Ignoranten, von denen einer mehr verdirbt als ein Halbdutzend brave Männer gut machen können” ${ }^{2}$. Wessenberg hat auch die Zeitschrift „Geistliche Monatsschrift” gegründet, wo er seine aufgeklärte Ideen nicht nur für Alumnen, sondern auch für alle Priester seiner Diözese erfasst hat ${ }^{53}$.

2) Bildung der Gläubigen

Bildung war für Ignaz Heinrich von Wessenberg eine gute Unterweisung im christlichen Glauben und auch eine Stärkung der Kirchlichkeit und Moralität der

49 D. Rimmale, Der Bildung des Voskes..., op. cit., S. 43-44.

50 S. Schmid, Der Priester..., op. cit., S. 38-39.

51 K.H. Braun, Den Glauben bewusster..., op. cit., S. 21-22.

52 F.X. Bischof, Die Bemühungen ..., op. cit., S. 104.

53 K.H. Braun, Ganz diesem Beruf..., op. cit., S. 232. 
Gläubigen ${ }^{54}$. Diese vollzieht sich vor allem durch ihre Teilnahme an verschiedenen Gottesdiensten. „Es war damals üblich gewesen, dass priesterliche Handlungen am Altar vom religiösen Verhalten der Gläubigen im Kirchenraum vollständig abgekoppelt waren. Während der zelebrierende Priester die lateinischen Texte des Missale Romanum leise oder halblaut murmelnd las, pflegte die Gemeinde in einem überbietenden Lautpegel den Rosenkranz zu beten - ohne Rücksicht darauf, was am Altar geschah" ${ }^{55}$. Er regte sich auf über gedanken - und lieblose Zelebrationen, die manchmal sogar gleichbedeutend mit Säkularisation waren. Die Liturgie sollte den Gläubigen eine innere Beziehung zu Gott ermöglichen. Menschen sollten in der Zeit des Gottesdienstes eine Einladung zur Mitarbeit mit göttlicher Gnade bekommen ${ }^{56}$. „Für Wessenberg war es eine »heilige Pflicht«, denkend Gott und die Welt zu verbinden" ${ }^{57}$. Darin bestehe »die Weisheit des Christentums «, dass im denkenden Erfassen der vielfältigen Beziehung zwischen dem himmlischen Jenseits und dem irdischen Diesseits unser Vertrauen und Zuversicht zum heimlichen Vater wachsen kann. Seiner Meinung nach müssten solche Praktiken wie das Rosenkranzbeten während der heiligen Messe reduziert werden. Er betrachtete das als einen gedankenlosen Mechanismus, der die Beziehung zu Gott nicht stärkt, sondern sogar die geistig-geistliche Begegnung verhindert und abbaut ${ }^{58}$.

Was den Bereich der Liturgie betrifft, meinte er auch, dass nicht nur die Epistel und das Evangelium in deutscher Sprache proklamiert werden sollten, sondern auch Gloria, Credo und die wesentlichen Gebetselemente der Opfergabe und Kommunion. Um den Gläubigen Gott zu nähern, hat Wessenberg manche Gebete in eine poetische, das heißt durch Rhythmus und Reim strukturierte Fassung, gekleidet. Er hat sich auch nicht gescheut, den lateinischen Grundtext zu variieren. Die Gebetstexte zum Ordo ergänzte Wessenberg mit Bezugstexten zum jeweiligen Tag oder Zeitraum, wie z.B. Sonntage, Advent, Weihnachten, Ostern, Pfingsten, Karwoche, Fronleichnam, Himmelfahrt, Erntedank, Kirchweihe, Allerheiligen, Allerseelen, sowie mit Texten für diverse Apostels- und Märtyrermessen und vor allem für die Marienfeiertage. Des Weiteren war Wessenberg auch Autor zahlreicher Kirchenlieder. Er sagte, dass der Volksgesang zu den besonders wirksamen Förderungsmitteln der geistigen und gemütlichen Veredlung eines Volkes gehörte ${ }^{59}$. $\mathrm{Zu}$ den Mechanismen, die einen verheerenden Einfluss auf die Beziehung zu Gott hatten, zählte

${ }_{54}$ M. Thurau, Der Mystizismus ist ein Akt der Verzweiflung. Theologische Reflexion zu mystischen Phänomenen des 19. Jahrhunderts, in: Mystikerinnen der Neuzeit und Gegenwart, Hg. A. Middelbeck-Varwick, M. Thurau, Frankfurt am Main 2009, S. 49.

55 K. Oettinger, Der Dichter: Die Lieder und Hymnen Ignaz Heinrich von Wessenbergs, in: Bildung bei Ignaz Heinrich von Wessenberg (1774-1860), Hg. K.H. Braun, Freiburg im Breisgau 2014, S. 71.

56 K.H. Braun, Den Glauben bewusster..., op. cit., S. 22.

57 K.H. Braun, Der Gottsucher..., op. cit., S. 28.

58 K.H. Braun, Der Gottsucher..., op. cit., S. 29-34.

59 K. Oettinger, Der Dichter ..., op. cit., S. 71-75. 
Wessenberg auch die Wallfahrten, die er nur für eine Art Ausflug hielt, womit auch sehr häufig die Beichte verbunden war. Diese sollte viel mehr als eine regelmäßige individuelle Begleitung auf diesem bewussten Lernweg verstanden werden als ein Teil der Wallfahrten ${ }^{60}$. Ferner, um die geistliche Situation der Gläubigen zu verbessern, hat Wessenberg vorgeschlagen, die Zeit der Erstkommunionsvorbereitung zu verlängern. Er wollte, dass die Kinder besser verstehen, wozu sie berufen sind. Des Weiteren sollte laut Wessenberg die Predigt des Priesters während der heiligen Messe eine Erklärung, Belehrung und Motivation für die Gläubigen sein ${ }^{61}$.

Eine weitere wichtige Initiative, neben der Priesterbildung und den liturgischen Reformen, die auch zur Bildung der Gesellschaft beitragen sollten, war die Gründung der sogenannten Lesegesellschaften. „Hier sollten Schriften angeschafft und im Klerus eines Landkapitels verbreitet werden, die nicht vom jedem gekauft werden können [...]. Wessenberg schlägt drei Kategorien vor, nämlich Monats - oder Quartalsschriften mit Aufsätzen mit Literaturhinweisen, Fachbücher verschiedener theologischer und anderer Wissenschaften und Werke, die der Geistes- und Herzbildung dienen" ${ }^{2}$. Sie sollten kostenlos verbreitet und dann den Bibliotheken weitergegeben werden; somit sollten Bibliotheken für Jedermann entstehen und zugänglich sein. Jedoch ist diese Idee leider gescheitert. Darüber hinaus ist wichtig zu erwähnen, dass Wessenberg die besondere Form der Kommunikation und Bildung des Glaubens in der Beschäftigung mit den Kunstwerken gesehen hat. Ein von den Zimmern in seinem Haus war mit mehreren Kunstwerken »bebildet«. In dieser Sammlung sah er auch pädagogische und katechetische Implikationen ${ }^{63}$. Seine private Kunstgalerie öffnete er jedem, der sie sehen und sich dadurch bilden wollte, sogar dann, wenn er selbst abwesend war. Wessenberg war der Überzeugung, dass „die Menschheit sich im Laufe der Geschichte gerade durch die Kunst aufwärts entwickle und hilft bei der Entwicklung der Vollkommenheit" ${ }^{\prime 64}$. Er hat auch gesagt, dass die wahre Kunst eine Dienerin des Glaubens ist ${ }^{65}$, weil durch die Sinne das Heilige in die Herzen dringe ${ }^{66}$.

Ignaz Heinrich von Wessenberg war sicherlich jener Mann und Priester, der keine Angst hatte, seine Augen zu öffnen, um die Probleme der Menschen zu beobachten, die in wirklich schwierigen Zeiten lebten. Er und seine Genossen mussten unter anderem Expansionen der Französischen Revolution, Flucht vor dem Krieg, das Ende des Heiligen Römischen Reiches erleben ${ }^{67}$. In diesen schwierigen Zeiten

\footnotetext{
60 S. Schmid, Der Priester..., op. cit., S. 39.

${ }^{61}$ K.H. Braun, Den Glauben bewusster..., op. cit., S. 22.

62 D. Rimmale, Der Bildung des Voskes..., op. cit., S. 44

63 M. Bangert, Der Kunstliebhaber und Kunsttheoretiker in: Bildung bei Ignaz Heinrich von Wessenberg (1774-1860), Hg. K.H. Braun, Freiburg im Breisgau 2014, S. 92-93.

${ }_{64}$ M. Bangert, Der Kunstliebhaber und Kunsttheoretiker ..., op. cit., S. 99.

65 M. Bangert, Der Kunstliebhaber und Kunsttheoretiker ..., op. cit., S.117.

${ }_{66}$ M. Bangert, Bild und Glaube ..., op. cit., S. 300.

${ }^{67}$ K.H. Braun, Einleitung, in: Bildung bei Ignaz Heinrich von Wessenberg (1774-1860), Hg. K.H. Braun, Freiburg im Breisgau 2014, S. 9.
} 
versuchte Wessenberg, auf seine Art und Weise Gott zu finden und den Menschen mitzuteilen. Dies war eine Art der josephinistischen Aufklärung. Sein Seelsorgekonzept, das von der rationalen Annäherung zu Gott handelte, war seine eigene Antwort auf die damaligen großen Probleme und die schwierige Lage der Menschen und der Kirche $^{68}$.

\section{Bibliographie}

\section{Studien:}

Allmayer-Beck J.Ch., Colloredo-Waldsee, Hieronymus Grafvon, in: Neue Deutsche Biographie, Bd. 3, Berlin 1957, S. 327.

Aurnhammer A., Besslich B., Freiburg als Zentrum der südwestdeutschen katholischen Aufklärung zwischen Josephinismus und Frühliberalismus, in: Der Ort und das Ereignis: die Kulturzentren in der europäischen Geschichte, Hg. A. Venture1li, Freiburg 2002, S. 121-145.

Bangert M., Bild und Glaube. Ästhetik und Siritualität bei Ignaz Heinrich von Wessenberg (1774-1860), Stuttgart 2009.

Bangert M., Der Kunstliebhaber und Kunsttheoretiker in: Bildung bei Ignaz Heinrich von Wessenberg (1774-1860), Hg. K.H. Braun, Freiburg im Breisgau 2014, S. 89-131.

Bautz F.W., Colloredo Hieronymus Graf von, in: Biographisch-Bibliographisches Kirchenlexikon, Bd. 1, Hamm 1990, Sp. 1098.

Bischof X. F., »Die Einheit der Nationalkirche schien mir zunächst das Wesentliche, wenn sich das religiös-kirchliche Leben Unseres Volkes haben und gedeihlich sich entwickeln soll«. Wessenberg auf dem Wiener Kongress, in: Der Wiener Kngress - eine kirchenpolitische Zänsur?, Hg. H. Duchhardt, J. Wischmeyer, Göttingen 2013, S. 99-112.

Braun K.H., (Liberale) Kritik am Mönchtum, in: Mittelalterliches Mönchtum in der Moderne? Die Neugründigung der Benediktionerabtei Beuron 1863 und deren kulturelle Ausstrahlung im 19. und 20. Jahreshundert, Hg. W. Schöntag, Stuttgart 2015, S. 83-100.

Braun K.H., Den Glauben bewusster leben: vor 150 starb der Aufklärer Ignaz Heinrich von Wessenberg, „Konradsblatt” 2010, Nr. 32, S. 20-23.

Braun K.H., Der Gottsucher: Wie stellt er die Gottesfrage?, in: Bildung bei Ignaz Heinrich von Wessenberg (1774-1860), Hg. K.H. Braun, Freiburg im Breisgau 2014, S. 23-35.

Braun K.H., Die Lebensgeister der Kirche, Glaube und Liebe, bedürfen, um stets ungeschwächt und ungestört zu wirken, der beständigen Erneuerung. Zum Kirchen-

${ }^{68}$ K.H. Braun, Der Gottsucher..., op. cit., S. 23. 
bild Ignaz Heinrich von Wessenbergs, in: Kontinuität und Innovation um 1803. Säkularisation als Transformationsprozeß. Kirche, Theologie, Kultur, Staat, Hg. R. Decot, Mainz 2005, S. 21-38.

Braun K.H., Ganz diesem Beruf zu leben... mein volles Kraftmaß zu widmen..., in: Glauben gestalten, Glaubensgestalten: mit Robert Zollitsch auf dem Weg; zum 70. Geburtstag des Erzbischofs von Freiburg und Vorsitzenden der Deutschen Bischofskonferenz, Hg. F. Deck, Freiburg 2008, S. 226-235.

Braun K.H., Kirche im liberalen Bürgerstaat Das Erzbistum von der Mitte des 19. Jahrhunderts bis zum Ende der Monarchie 1918, in: Geschichte der Erzdiözese Freiburg Freiburg, Bd. 1, Hg. H. Smolinsky, Freiburg im Breisgau 2008, S. 121210.

Braun K.H., Wessenberg, Ignaz Heinrich von (1774-1860), in: Die Bischöfe der deutschsprachigen Länder, 1785/1803 bis 1945. Ein biographisches Lexikon, Hg. E. Gatz, Berlin 1893, S. 808-812.

Braun K.H., Wieder die Säkularisation. Obrigkeitliche Steuerungen von Religiosität innerhalb»josephinischer « Kirchenordnungen, in: Aufmerksame Solidartät: Festschrift für Bischof Maximilian Aichern zum 70. Geburtstag, Hg. P. Hofer, Regensburg 2002, S. 13-25.

Ferihumer H., Gall Joseph Anton, in: Neue Deutsche Biographie, Bd. 6, Berlin 1964, S. 42.

Leidl A., Leopold Ernst Kardinal von Firmian, „Ostbairische Grenzmarken. Passauer Jahrbuch für Geschichte, Kunst und Volkskunde" 1971, Bd. 13, S. 5-26.

Leidl A., Leopold Ernst von Firmian, in: Neue Deutsche Biographie, Bd. 14, Berlin 1985, S. 293.

Nörber C., Wessenberg Ignaz Heinrich von, http://kathenzyklo.bplaced.net/

artikel.php?artikel=wessenberg [Zugang: 25 X 2019].

Oettinger K., Der Dichter: Die Lieder und Hymnen Ignaz Heinrich von Wessenbergs, in: Bildung bei Ignaz Heinrich von Wessenberg (1774-1860), Hg. K.H. Braun, Freiburg im Breisgau 2014, S. 71-87.

Reusch F.H., Sailer Johann Michael von, in: Allgemeine Deutsche Biographie, Bd. 30, Leipzig 1890, S. 178-192.

Rimmale D., Der Bildung des Voskes: Die Bildungspolitik Wessenbergs, in: Bildung bei Ignaz Heinrich von Wessenberg (1774-1860), Hg. K.H. Braun, Freiburg im Breisgau 2014, S. 43-49.

Ruppert S., Kirchenrecht und Kulturkampf. Historische Legitimation, politische Mitwirkung und wissenschaftliche Begleitung durch die Schule Emil Ludwig Richters, Tübingen 2002.

Schmid S., Der Priester: »In der Mitte zwischen Gott und den Menschen, wischen der Gemeinde und dem Altar«, in: Bildung bei Ignaz Heinrich von Wessenberg 
(1774-1860), Hg. K.H. Braun, Freiburg im Breisgau 2014, S. 37-41.

Schmidt B., Die Konzilien und der Papst. Von Pisa (1409) bis zum Zweiten Vatikanischen Konzil (1962-65), Freiburg im Breisgau 2013.

Thurau M., Der Mystizismus ist ein Akt der Verzweiflung. Theologische Reflexion zu mystischen Phänomenen des 19. Jahrhunderts, in: Mystikerinnen der Neuzeit und Gegenwart, Hg. A. Middelbeck-Varwick, M. Thurau, Frankfurt am Main 2009, S. 37-74.

Werner K., Gall Joseph Anton, in: Allgemeine Deutsche Biographie, Bd. 8, Leipzig 1878, S. 317.

Wessenberg I.H., Die Eintracht zwischen Kirche und Staat, Hg. J. Beck, Aarau 1869.

Wessenberg I.H., Unveräffentlichte Manuskripte und Brieffe I/1, Hg. K. Aland, V. Müller, Freiburg-Basel-Wien 1968.

Xaver F.B., Die Bemühungen des Konstanzer Generalvikars Ignaz Heinrich von Wessenberg um die Priesterfortbildung, https://mthz.ub.unimuenchen.de/MThZ/ article/download/

1995H1S99-117/3938/ [Zugang: 27 X 2018].

\section{Zarys koncepcji duszpasterskiej Ignacego Henryka von Wessenberga}

\section{Streszczenie}

Artykuł jest próbą przedstawienia zarysu koncepcji pastoralno-duszpasterskiej Ignacego Henryka von Wessenberga, który odegrał istotną rolę w budowaniu struktur administracyjno-duszpasterskich tworzonej właśnie diecezji Konstanz. Von Wessenberg był wikariuszem generalnym i administratorem tego biskupstwa. Jego poglądy wskazywały na chęć utworzenia w Niemczech Kościoła episkopalnego, kierowanego wprawdzie przez prymasa, ale opartego na ustroju Kościołów krajowych przenikniętych ideami austriackiego oświecenia. Gruntowną reformę życia duchowego chciał on zaprowadzić przez odnowę liturgii oraz odpowiednie kształcenie kleru.

Publikacja nakreśla czynniki mające wpływ najpierw na rozwój osobowości Wessenberga, a następnie na jego poglądy dotyczące Kościoła oraz duszpasterstwa. Akcentuje rolę ojca i wpływ ,józefińskich” biskupów, których poglądy bardzo wyraźnie odcisnęły się w późniejszych działaniach pastoralnych Wessenberga. Następnie tekst przedstawia jego zapatrywania na dogmatykę, eklezjologię oraz opartą o nie koncepcję duszpasterstwa, która najlepiej wyraziła się w procesie formacji duchowieństwa, kleru i wiernych.

Słowa klucze: duszpasterstwo, Konstanz, kształcenie, oświecenie, reforma, Wessenberg 


\title{
An Outline of Ignaz Heinrich von Wessenberg's Pastoral Vision
}

\begin{abstract}
Summary
This article attempts to present the pastoral vision of German priest Ignaz Heinrich von Wessenberg, who played an important role in the creation of the Konstanz diocese and the formation of this diocese's pastoral and administrative structures. Von Wessenberg was a vicar-general and an administrator of this new ecclesiastical unit. His activities reflected his idea of establishing an episcopal Church in Germany, headed by a primate, modelled upon national churches in accordance with ideas of Austrian Enlightenment. He was also actively promoting a thorough reform of spiritual life, by the means of liturgy and appropriate clerical formation.

This article first presents factors that influenced the personality of Wessenberg and his views on the Church and pastoral care. It emphasises the role of his father and ideas of "Josephinist" bishops that left a distinct mark on his future pastoral work. Next it presents Wessenberg's views concerning ecclesiology and dogmatic theology and the impact these views had on his idea of pastoral care, which found its best expression in the formation processes of the clergy and laity that he supervised.
\end{abstract}

Key words: pastoral care, Konstanz, formation, enlightenment, reform, Wessenberg 